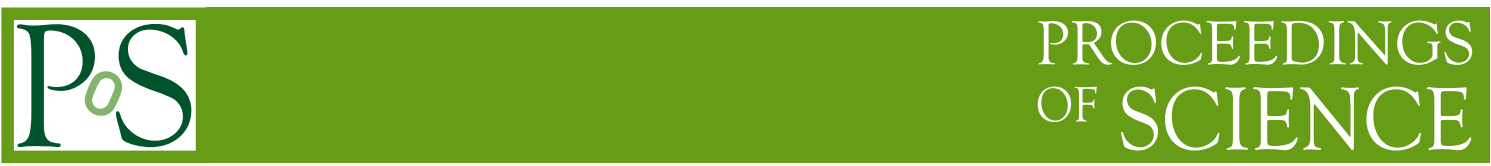

\title{
Neutrino flavor-, helicity-, and pair oscillations
}

\section{A. Kartavtsev*}

Max-Planck-Institut für Physik, Föhringer Ring 6, 80805 München, Germany

E-mail: alexander.kartavtsev@mpp.mpg.de

\section{G. Raffelt}

Max-Planck-Institut für Physik, Föhringer Ring 6, 80805 München, Germany

E-mail: raffelt@mpp.mpg.de

\section{H. Vogel}

Max-Planck-Institut für Physik, Föhringer Ring 6, 80805 München, Germany

E-mail: hvogel @mpp.mpg • de

\begin{abstract}
Neutrinos propagating in media undergo flavor and helicity oscillations, where helicity transitions are instigated both by electromagnetic fields and matter currents. In addition, it has been shown that correlations between neutrinos and antineutrinos of opposite momentum can build up in anisotropic media. Here, we discuss the physical meaning of the helicity and neutrinoantineutrino correlations, their potential impact on the supernova dynamics, the conditions under which they can be generated, and present an order-of-magnitude estimate of their size. Even in the vicinity of the core both are typically very small, of the order of $10^{-11}$. However, in the presence of strong matter and neutrino currents parallel to the neutrino momentum, the helicity coherence can be resonantly enhanced and affect the supernovae dynamics. On the other hand, a resonant enhancement of the neutrino-antineutrino coherence does not seem to be possible.
\end{abstract}

18th International Conference From the Planck Scale to the Electroweak Scale 25-29 May 2015

Ioannina, Greece

\footnotetext{
*Speaker.
} 


\section{Introduction}

Supernovae explosions are among the most fascinating and beautiful events in the Universe. The earliest recorded supernova, $S N$ 185, was viewed by Chinese astronomers in 185 AD. Supernovae $S N 1572$ and $S N$ 1604, the latest to be observed with the naked eye in the Milky Way galaxy, had notable effects on the development of astronomy in Europe. Since the invention of the telescope, the field of supernova discovery has extended to other galaxies, starting with the 1885 observation of a supernova in the Andromeda galaxy. Roughly a century later, another supernova, SN 1987 A, was, for the first time in the history of humankind, not only observed in photons but also in neutrinos.

Neutrinos play a crucial role in the explosion of a core-collapse supernova. The explosion produces about $10^{57}$ neutrinos with $10 \mathrm{MeV}$ average energy, and these take away $99 \%$ of the gravitational energy. Because of their very weak interaction with usual matter, neutrinos are capable of leaving the supernova. The neutrino emission results in the neutronization of the supernova core, leading to a decrease of the pressure in the core and its rapid implosion. With the implosion the internal pressure inside the core starts to increase dramatically and the collapse abruptly halts giving place to a violent reexpansion. A strong shock front begins to plow through material that is still falling inward at roughly sixty thousand kilometers per second. The core bounce accelerates the infalling material but then quickly stalls. It is the escaping neutrinos that pump energy into the shock wave and rescue the explosion. Neutrino interactions with the matter of the outer layers are also believed to be responsible for the production of the heavy elements in the Universe.

In numerical simulations, supernovae currently explode in 2D and first examples of exploding 3D models have recently appeared in the literature [1,2]. Given the importance of neutrinos for the supernova physics, the phenomenon of neutrino oscillations plays an important role in the simulations of the explosion. Neutrino flavor oscillations in supernovae have been investigated in great detail. Because of the huge neutrino density neutrino-neutrino interactions are not negligible, which makes the evolution equations non-linear and leads to unstable run-away solutions. The observation that even very small effects can eventually grow large because of the non-linearity of the neutrino evolution has recently sourced interest to two additional types of coherence, helicity coherence and neutrino-antineutrino coherence. Here we explain what these are, what impact they might have on the supernova physics, how they are produced in a supernova environment, and present an order-of-magnitude estimate of their size. We argue that while helicity coherence can in principle be resonantly enhanced and affect the supernova dynamics, the neutrino-antineutrino coherence is very small and can be neglected.

\section{Flavor coherence and oscillations}

Before going to the unknown terrain of helicity and neutrino-antineutrino correlations let us briefly review the well-known phenomenon of flavor coherence, mainly to illustrate two things. First, what coherence means in the context of neutrino physics. Second, how the coherence can be described at the technical level.

In a decay of $W$-bosons, a neutrino is produced in a flavor eigenstate, and its flavor is tagged by the produced charged lepton, be it the electron, muon, or tauon. A neutrino flavor eigenstate 
is a linear superposition of its mass eigenstates. This leads to the beautiful phenomenon of neutrino oscillations. As the neutrino propagates from the production point to the detector, the phases between the mass eigenstates change and so changes the probability to observe a neutrino of a particular flavor. In practice, the description of the flavor oscillations typically relies on the use of the Schrödinger equation. This approach is simple and intuitive, and it is efficient for studying neutrino propagation in the Sun or neutrino oscillations in terrestrial experiments. In a supernova environment, however, it does not grasp another important aspect of the problem, namely that in a dense supernova medium the neutrinos can undergo scattering on matter particles as well as on other neutrinos. The scattering processes can be described by the Boltzmann equation, but the Boltzmann equation does not take into account coherence effects.

This was the dilemma neutrino physicists faced two decades ago, until Georg Raffelt and Günter Sigl developed density matrix equations that could simultaneously take into account coherence and scattering effects [3]

$$
\dot{\rho}=-i[\mathrm{H}, \rho]+C[\rho],
$$

In the language of density matrices flavor coherence means that the density matrix in the flavor basis has non-zero off-diagonal elements,

$$
\rho=\left(\begin{array}{ll}
\rho_{e e} & \rho_{e \mu} \\
\rho_{\mu e} & \rho_{\mu \mu}
\end{array}\right) .
$$

The oscillations are induced by the commutator of the density matrix with the Hamiltonian matrix $\mathrm{H}$, which is not diagonal in the flavor basis. The larger are the off-diagonal elements of the Hamiltonian, the faster the coherence builds up and the larger is the magnitude of the produced coherence. Finally, the scattering effects are described by the collision term $C[\rho]$.

\section{Helicity coherence and oscillations}

Now that we have introduced the concept of the density matrix, we can jump directly to helicity oscillations recently studied in refs. [4, 5] using the Kadanoff-Baym formalism and in refs. [6, 7] using the density matrix formalism.

Helicity is the projection of the neutrino spin on its momentum. A massless neutrino can be in one of the two pure helicity states: its spin is either aligned with or against its momentum. A massive neutrino can be in a superposition of the two pure states, in such a case one can speak of helicity coherence. In the language of the density matrix, helicity coherence means that the density matrix in the mass basis has non-zero off-diagonal elements,

$$
\rho=\left(\begin{array}{c}
\rho_{--} \rho_{-+} \\
\rho_{+-} \rho_{++}
\end{array}\right) .
$$

A nontrivial property of helicity coherence is that it is not Lorentz invariant. By boosting in the direction opposite to the neutrino momentum one can flip the momentum direction and therefore the helicity. Similarly, by boosting in a direction orthogonal to the neutrino momentum we create a superposition of two helicity eigenstates and therefore create helicity coherence. This given, one 
should specify the reference frame, for example the rest frame of the supernova, to speak about a build up of helicity coherence.

What the helicity coherence does depends on the neutrino nature. If the neutrino is a Majorana particle then helicity coherence leads to a conversion of a neutrino into an antineutrino. This affects the neutron-to-proton ratio, which is the key determinant of nucleosynthesis. On the other hand, if the neutrino is a Dirac particle then helicity coherence converts an active neutrino into a sterile neutrino. This in turn affects the energy transfer to the outer layers. The impact of the latter effect depends on the oscillation length.

The next question is what is needed to create helicity coherence and how large it can be. Assume that a neutrino is produced in a helicity eigenstate and propagates in a homogeneous medium. It is known that the neutrino mass term flips the spin. But the mass term alone is not sufficient because the spin flip changes the total angular momentum of the system. In addition, we need a vector that is orthogonal to the neutrino momentum and breaks the axial symmetry of the system. In a supernova, such a vector can be provided by a matter current or by a magnetic field. Thus, we have two components of the effective Hamiltonian, the matter component and the magnetic component ${ }^{1}$

$$
\mathrm{H} \approx\left(\begin{array}{cc}
V^{0}-V_{\|} & \frac{m}{2 p} V_{\perp} \\
\frac{m}{2 p} V_{\perp} & 0
\end{array}\right)-\mu B\left(\begin{array}{ll}
0 & 1 \\
1 & 0
\end{array}\right),
$$

where $V^{0}$ is the potential induced by matter particles and neutrinos, whereas $V_{\|}$and $V_{\perp}$ are components of the matter and neutrino fluxes parallel and orthogonal to the neutrino momentum. Finally, $\mu$ is the neutrino magnetic dipole moment and $B$ is the magnetic field. Which of the contributions dominates? This depends on where in the supernova we are. Let us assume for definiteness that we are close to the neutrino-sphere which is a ball of roughly twenty kilometers in diameter in the heart of the supernova. In this region, the magnetic contribution is a few orders of magnitude smaller than the matter contribution. The natural order of magnitude for the produced helicity coherence is of the order of $10^{-11}$,

$$
\rho \sim\left(\begin{array}{cc}
1 & \frac{m}{2 p} \beta \\
\frac{m}{2 p} \beta & 0
\end{array}\right) \sim\left(\begin{array}{cc}
1 & 10^{-11} \\
10^{-11} & 0
\end{array}\right) .
$$

In other words, in the natural mixing regime the helicity coherence is likely too small to have any observable impact. This can be traced back to the smallness of the neutrino mass compared to the typical neutrino energies. The neutrinos are highly relativistic and it is very difficult to flip their spin.

But the situation is not hopeless, as has been pointed out in ref. [6]. The smallness of the produced helicity coherence can be traced back to the smallness of the off-diagonals of the Hamiltonian compared to its diagonals. The rotation angle needed to diagonalize this matrix is very small and so is the mixing between the helicity eigenstates. However, let us recall the MSW effect. If the difference of the diagonal elements vanishes, then there is a resonant enhancement of the mixing. Applied to helicity coherence, that means that, if there is a strong matter current parallel to the neutrino momentum, then the diagonals of the Hamiltonian vanish [see eq. (3.2)] and the build up

\footnotetext{
${ }^{1}$ The spin-flavor conversion induced by the magnetic field was first addressed in works of A. Studenikin and collaborators $[8,9]$.
} 
of the helicity coherence is resonantly enhanced. In this case, the effect may well be large and play a role in the supernova dynamics. The question is, of course, if this resonance condition is ever fulfilled in a supernova and if the transition is adiabatic. According to the estimates and numerical analysis performed in ref. [6] the former is the case, at least in or near the proto-neutron star in a core-collapse supernova, whereas the fulfillment of the adiabaticity condition is unclear.

Before moving to even more exotic neutrino-antineutrino oscillations let us summarize this section. First, helicity coherence means that the neutrino is in a superposition of its two helicity eigenstates. Second, the natural size of the induced helicity coherence is of the order of $10^{-11}$ and is likely negligibly small. And third, in certain regions of supernovae with strong matter currents an analogue of MSW effect can occur and the helicity correlations can become large.

\section{Neutrino-antineutrino coherence and oscillations}

We are now ready to move to neutrino-antineutrino coherence. This type of correlations has recently been "exported" to neutrino physics from solid state physics in refs. [7, 10, 11]. In solid state physics similar correlations are essential for the description of superconductors. In particle physics, on the other hand, they are very exotic. What does neutrino-antineutrino coherence mean in the context of neutrino physics? It is not, as the name misleadingly suggests, a correlation between a neutrino and antineutrino. Instead, it is a correlation between a vacuum state and a state with one neutrino and one antineutrino with opposite momenta. It is worth mentioning, that for producing neutrino-antineutrino coherence, we do not even need a neutrino mass - it arises already for a massless Weyl neutrino. In this case, neutrino-antineutrino coherence is parametrized by off-diagonal elements of the generalized density matrix

$$
\mathrm{R}=\left(\begin{array}{cc}
\rho & \kappa \\
\kappa^{\dagger} & 1-\bar{\rho}
\end{array}\right)
$$

What neutrino-antineutrino oscillations do follows from what neutrino-antineutrino coherence is. A nonzero particle-antiparticle coherence changes the definition of the true vacuum state and results in production of neutrino-antineutrino pairs with opposite momenta. Loosely speaking, the effect is similar to the Klein paradox. A neutrino beam enters a dense medium, neutrinoantineutrino coherence builds up, and a small beam of antineutrinos flowing in the opposite direction is generated. To make things even more confusing, even if initially there are no neutrinos or antineutrinos, in a dense medium a nonzero neutrino-antineutrino coherence is produced by the matter current. The generated neutrino-antineutrino coherence, in turn, leads to a production of neutrino-antineutrino pairs out of the vacuum. This can be seen as a shift of the Fermi sea and is somewhat similar to the sphaleron processes in the early Universe.

The next question is how the neutrino-antineutrino correlations build up and what we need to create them. Because we create a neutrino-antineutrino pair with opposite momenta, the total angular momentum of the system changes and we need a matter flow orthogonal to the neutrino momentum to break the axial symmetry of the system. This is the same condition as for helicity coherence. Therefore, for a massive neutrino, both helicity and neutrino-antineutrino coherencies are produced simultaneously. 
Knowing the corresponding effective Hamiltonian,

$$
\mathrm{H}=E\left(\begin{array}{cc}
1 & 0 \\
0 & -1
\end{array}\right)+\left(\begin{array}{cc}
V^{0}-V_{\|} & -V_{\perp} \\
-V_{\perp} & V^{0}+V_{\|}
\end{array}\right),
$$

we can estimate the size of the produced neutrino-antineutrino coherence. Its natural size is given by the mixing angle, that is by the angle of the unitary transformation that diagonalizes the Hamiltonian. Using $V_{\perp} \sim V^{0} \beta$ and substituting the typical supernovae densities and maximal matter flow velocities $\beta \sim 10^{-2}$ we again arrive (see ref. [12]) at a tiny number of the order of $10^{-11}$,

$$
\mathrm{R} \sim\left(\begin{array}{cc}
\rho & \frac{V^{0} \beta}{2 E} \\
\frac{V^{0} \beta}{2 E} & 1-\bar{\rho}
\end{array}\right) \sim\left(\begin{array}{cc}
\rho & 10^{-11} \\
10^{-11} & 1-\bar{\rho}
\end{array}\right) .
$$

As compared to the case of helicity coherence the neutrino mass in the mixing angle has been replaced by the neutrino potential, but numerically the latter lies in the same ballpark as the neutrino mass.

We can ask ourselves if there is a chance to get a resonant MSW-like enhancement, as it occurs for helicity correlations. Formally yes. If the component of the neutrino potential $V_{\|}$would be equal to the kinetic neutrino energy, then we would have a resonance. But in reality there are at least two reason why such a resonance should not be considered. First, a typical neutrino energy in a supernova is roughly $10-20 \mathrm{MeV}$. This is to be compared to the neutrino potential induced by the weak interactions, which is roughly $0.1 \mathrm{eV}$. There are eight to nine orders of magnitude difference and under no circumstances can the weak interactions become strong enough to bridge this huge gap. Apart from this numerical argument, there is also a conceptual one. A resonant enhancement of neutrino-antineutrino coherence would mean that the usual perturbative vacuum would no longer be an adequate expansion point and the usual perturbative definition of a particle would be rendered meaningless. From the practical point of view this means that neutrino-antineutrino correlations are always small.

This brings us to another intermediate summary. First, a nonzero neutrino-antineutrino coherence means that there is a superposition of vacuum and neutrino-antineutrino states. Second, natural size of the induced neutrino-antineutrino coherence is of the order of $10^{-11}$ and is likely negligibly small. And third, unlike for helicity coherence, neutrino-antineutrino coherence cannot be resonantly enhanced.

\section{Summary}

Neutrino flavor conversion is important in supernovae, yet a full understanding remains elusive, largely because of neutrino-neutrino refraction and concomitant self-induced flavor conversion, an effect caused by run-away modes of the interacting neutrino gas. The difficulties in developing a robust phenomenological understanding of even this relatively simple case explains the reluctance to add further complications. Here, we have addressed two additional types of neutrino oscillations, the helicity and neutrino-antineutrino ones, that have recently attracted a renewed interest. 
As the name suggests, helicity oscillations describe transitions between two helicity eigenstates of a massive neutrino. It is known, that a neutrino mass term flips the spin. However, this alone is not sufficient to instigate the helicity oscillations because the spin flip changes the total angular momentum. In addition, one needs a vector orthogonal to the neutrino momentum that breaks the axial symmetry of the system. In a supernova, such a vector can be provided by a matter current or by a magnetic field. For typical supernova parameters, the natural size of the induced helicity coherence is of the order of $10^{-11}$ and is likely negligibly small. However, in certain regions of a supernova with strong matter currents, an analogue of MSW effect can occur, and the helicity oscillations might potentially become important. Their impact on supernova physics depends on the neutrino nature. If the neutrino is a Majorana particle, then helicity oscillations lead to a conversion of a neutrino into an antineutrino, thereby affecting the neutron-to-proton ratio. On the other hand, if the neutrino is a Dirac particle then helicity oscillations convert an active neutrino into a sterile neutrino thus affecting the energy transfer to the outer layers.

Helicity oscillations can be complicated in detail, but they are conceptually straightforward. The situation with the neutrino-antineutrino oscillations is more complicated. In the simplest case of massless neutrinos, the correlations are between neutrinos and antineutrinos of opposite momenta, and the oscillations are between the vacuum state and the one filled with a neutrino and antineutrino. A creation of such a neutrino-antineutrino pair out of the vacuum violates the total angular momentum. Thus, similarly to helicity oscillations, to instigate the neutrino-antineutrino oscillations one needs a matter flow orthogonal to the neutrino momentum to break the axial symmetry of the system. For typical supernova parameters, the natural size of the induced neutrinoantineutrino coherence is again of the order of $10^{-11}$ and likely negligibly small. Furthermore, a resonant enhancement of neutrino-antineutrino oscillations cannot take place in a realistic supernova environment.

The ultimate ambition of fully understanding neutrino propagation in dense environments and strong magnetic fields requires a more complete development of its theoretical underpinnings. Our contribution is meant as a step towards this overall goal.

\section{Acknowledgments}

We would like to thank I. Izaguirre, S. Chakraborty, A. Dobrynina, and C. Volpe for fruitful discussions. We acknowledge partial support by the Deutsche Forschungsgemeinschaft (DFG) under Grant No. EXC-153 (Excellence Cluster "Universe"), and by the Research Executive Agency (REA) of the European Union under Grant No. PITN-GA-2011-289442 (FP7 Initial Training Network "Invisibles").

\section{References}

[1] A. Mezzacappa, S. W. Bruenn, E. J. Lentz, W. R. Hix, O. E. B. Messer, J. A. Harris, E. J. Lingerfelt, E. Endeve, K. N. Yakunin, J. M. Blondin, and P. Marronetti, Two- and Three-Dimensional Multi-Physics Simulations of Core Collapse Supernovae: A Brief Status Report and Summary of Results from the "Oak Ridge" Group, arXiv:1405.7075. 
[2] T. Melson, H.-T. Janka, R. Bollig, F. Hanke, A. Marek, and B. Muller, Neutrino-driven Explosion of a 20 Solar-mass Star in Three Dimensions Enabled by Strange-quark Contributions to NeutrinoâĂŞnucleon Scattering, Astrophys. J. 808 (2015), no. 2 L42, [arXiv: 1504.07631$].$

[3] G. Sigl and G. Raffelt, General kinetic description of relativistic mixed neutrinos, Nucl. Phys. B 406 (1993) 423.

[4] A. Vlasenko, G. M. Fuller, and V. Cirigliano, Neutrino quantum kinetics, Phys. Rev. D 89 (2014) 105004, [arXiv:1309.2628].

[5] V. Cirigliano, G. M. Fuller, and A. Vlasenko, A New Spin on Neutrino Quantum Kinetics, Phys. Lett. B747 (2015) 27-35, [arXiv: 1406.5558 ].

[6] A. Vlasenko, G. M. Fuller, and V. Cirigliano, Prospects for neutrino-antineutrino transformation in astrophysical environments, arXiv:1406.6724.

[7] J. Serreau and C. Volpe, Neutrino-antineutrino correlations in dense anisotropic media, Phys. Rev. D 90 (2014) 125040, [arXiv:1409.3591].

[8] A. M. Egorov, A. E. Lobanov, and A. I. Studenikin, Neutrino oscillations in electromagnetic fields, Phys. Lett. B 491 (2000) 137, [hep-ph/ 9910476 ].

[9] A. Studenikin, Neutrinos in electromagnetic fields and moving media, Phys.Atom.Nucl. 67 (2004) 993-1002.

[10] C. Volpe, D. Väänänen, and C. Espinoza, Extended evolution equations for neutrino propagation in astrophysical and cosmological environments, Phys. Rev. D 87 (2013) 113010, [arXiv:1302.2374].

[11] D. Väänänen and C. Volpe, Linearizing neutrino evolution equations including neutrino-antineutrino pairing correlations, Phys. Rev. D 88 (2013) 065003, [arXiv:1306.6372].

[12] A. Kartavtsev, G. Raffelt, and H. Vogel, Neutrino propagation in media: Flavor-, helicity-, and pair correlations, Phys. Rev. D91 (2015), no. 12 125020, [arXiv: 1504.03230$].$ 